\title{
Influence of Timing in Introducing Teacher Performance Evaluation on Effective Outcomes: The Case of One Education District in Zimbabwe
}

\author{
Belinda R. Musodza \\ Department of Continuing Professional Teacher Development, \\ Faculty of Educational Sciences, \\ Walter Sisulu University, South Africa \\ Tawanda Runhare \\ Department of Foundations of Education, \\ School of Education, University of Venda, South Africa \\ Mamotena Mpeta \\ Department of Professional Studies, \\ School of Education, University of Venda, South Africa \\ Elphinah N. Cishe \\ Department of Continuing Professional Teacher Development, \\ Faculty of Educational Sciences, \\ Walter Sisulu University, South Africa
}

DOI: https://doi.org/10.36941/ajis-202o-0108

\begin{abstract}
This paper reports on a study that assessed how timing of introducing teacher performance evaluation in one district in Zimbabwe influenced the achievement of the desired outcomes. The research was premised on the pragmatic research paradigm and underpinned by the Readiness Assessment, Design, Process, Significance (RADPS) conceptual framework. The convergent parallel mixed method design was employed for data gathering and analysis from randomly selected 292 teachers for the quantitative strand and 12 purposively selected teachers for the qualitative strand. Descriptive statistics and inferential statistics (Analysis of Variance (ANOVA) and Beta coefficient tests) were used to analyse quantitative data and ATLAS ti. 8 generated quotations and networks that summarised the narrative views of teachers on the timing of introducing the performance evaluation system. The ANOVA result (o.ooo) indicated that there was a significant relationship between the timing of introducing performance evaluation and its effectiveness and the Beta coefficient value (0.213) indicated a strong influence of timing of introduction on effectiveness of performance evaluation. The quantitative results were corroborated by findings from interviews, which indicated that the system had been imposed, had inadequate budgetary support, no piloting prior to implementation, inadequate pre-implementation training and marketing. These resultantly led to limited buyin by stakeholders and a negative impact on the effectiveness of the whole performance evaluation system. Based on the findings, we concluded that due to its mistimed introduction, the performance evaluation system was ineffective in achieving its educational objectives in the Zimbabwean district where the study was conducted.
\end{abstract}

Keywords: effectiveness, institutional capacity, performance evaluation, readiness assessment, teacher performance, timing 


\section{Introduction}

There has been greater demand for accountability, transparency and results- based culture from governments globally (United Nations Educational, Scientific and Cultural Organisation (UNESCO), 2015; Kusek \& Rist, 2004). More communities are holding their governments to account and demanding services that are responsive to societal needs. One sure way of transforming and becoming accountable to communities served is by introducing performance management systems in general and evaluation systems in particular (Ochurub, Bussin \& Goosen, 2012). Performance management systems are an assortment of activities that are designed to maximise individual performance in order to achieve organisational goals while performance evaluation involves the measurement of employee performance against set targets within a given time period to inform decision making (Dorsey \& Mueller-Hanson, n.d).

The education sector, through the schools, has not been spared by the wide calls for accountability and results. Schools are mandated to provide a quality educational experience and learner achievement (Elliot, 2015). Teacher quality has been identified as any school's key resource in achieving the mandate of educating learners effectively (Darling-Hammond, 200o; Looney, 2011; Stronge \& Tucker, 2003; The New Teacher Project (TNTP), 2010; Sanders, Wright, \& Horn, 1997). Research suggests that effective teaching can only be assured by evaluating teachers (Shinkfield \& Stufflebeam, 1995). In response to the call for instructional quality, many countries, including Zimbabwe, have set up some form of teacher performance evaluation.

Zimbabwe has undergone a series of reforms intended to improve the civil service in general since its birth in 1980. These include the Public Finance Management System, Mission Statements, Clients Charters and Performance Management System before introducing the Results Based Management which is currently in use, and is the focus of this paper (Matunhu \& Matunhu, 2014; Zvavahera, 2014). Result Based Management (RBM) is an administrative tool that assists to track progress and demonstrate the outcomes and impacts of a policy (Kusek \& Rist, 2004:4). RBM is also defined as a management strategy which requires all stakeholders to contribute through processes or services, either directly or indirectly to the achievement of target results (UNDG, 2011). RBM reports on actual outcomes against expected results (Mayne, 2007). RBM extends beyond results and helps to inform future decision making by seeking for explanations for both successes and failures thereby highlighting the lessons learnt (Kusek \& Rist, 2004).

RBM was introduced as a new appraisal system in Zimbabwe in 2005. The aim of performance management is to improve the quality of service that is provided through improved worker performance (Ochurub et al., 2012). It would, however, seem like RBM has not brought the expected educational quality imperatives in terms of improved teaching and learning (Gutuza, 2016), hence the justification for this study to determine the influence of timing in introducing the evaluation system on effective outcomes. Research has shown that the success of performance evaluation systems is hinged on the presence of certain pre-existing conditions and attitudes such as appropriate organisational culture, a legal framework, creating demand, budgetary support, political will, and making the change process participatory and inclusive (Kusek \& Rist, 2004; Ochurub et al., 2012; Verspoor, 2004). This study was conducted on the background of scepticism, inadequate political will and pre-implementation planning for RBM in Kwekwe district. The aim was to investigate the preparedness of the district for the inception of the RBM as a teacher performance evaluation system through a process coined 'Readiness Assessment' (Kusek \& Rist, 2004). The study, thus, sought to measure and explain the appropriateness of the timing of introduction of the RBM as a teacher appraisal system in the district from the perspectives of teachers, heads of subject departments and school principals. This was done by ascertaining the existence of certain pre-conditions and attitudes prior to the introduction of RBM and establishing how their existence or absence could have impacted on the effectiveness of RBM as a performance evaluation system. This assessment was informed by the Readiness Assessment, Design, Process, Significance (RADPS) standards (Kusek \& Rist, 2004; Santiago \& Benavides, 2009; Shinkfield \& Stufflebeam, 1995; Stronge, 1995; The New Teacher Project (TNTP), 2010). 


\section{Conceptual Framework and Literature Survey}

This study was underpinned by a conceptual framework derived from literature, theories, beliefs and personal experiences of the researchers. The conceptual framework explains what we thought influences and affects the phenomenon under study. The concepts of the framework were borrowed from sources that include works by Kusek and Rist (2004), Santiago and Benavides (2009), Shinkfield and Stufflebeam (1995), Stronge (1995), and TNTP (2010) and elements from the Systems, Goal Setting and Expectancy theories. The conceptual framework constitutes four components namely Readiness Assessment, Design, Process and Significance (RADPS). This paper however, confines itself to the Readiness Assessment aspect of the framework as dictated by the scope of its discussion which relates to the timing of introducing RBM in Kwekwe district of Zimbabwe.

\subsection{Conceptualising readiness assessment}

According to Kusek and Rist (2004), 'readiness assessment' is a provision of an analysis framework to ascertain institutional preparedness to monitor and evaluate employee performance against a performance framework. Readiness assessment involves the systematic analysis of an organisation's ability to undergo a transformational process or change, by identifying potential challenges such as lack of stakeholder buy-in, political commitment, adequate resources, and legal statute to support the intended new procedure (Ochurub et al., 2012). For the purpose of this paper, we thus define readiness assessment as a process of establishing the preparedness of an organisation to embark on a new intervention by ascertaining the existence of pre-conditions like institutional capacity, organisational culture, political commitment, and demand.

\subsection{Purpose of readiness assessment}

The main purpose of conducting readiness assessment is to determine potential barriers to the successful implementation of an initiative, in this case, a performance evaluation system. The process facilitates a deep understanding of the identified barriers and provokes establishment of strategies to overcome the challenges (HSRA, n.d). The argument is that, readiness assessment facilitates the early indication of what to anticipate ahead before rolling out the new intervention (Ochurub et al., 2012). Readiness assessment is assumed to assist in the establishment of institutional preparedness for building, using and sustaining a new performance evaluation system, thereby increasing the likelihood of its success and effectiveness (Kusek \& Rist, 2004; Weiner, 2009). It allows the identification of gaps and provides an opportunity to address them before or during implementation which in turn enhances the feasibility of the planned intervention. A readiness assessment process is thus like the foundation of a building whose support is critical for all that is above it even though it is hidden underground. In the case of a performance evaluation system, readiness assessment helps to create a valuable awareness of the institutional capacity and its political willingness to monitor and evaluate its goals and objectives (Kusek \& Rist, 2004). The Readiness Assessment component of the RADPS conceptual framework is suitable for this study as it gives a detailed outline of factors that can affect organisational readiness for change. This facilitated a thorough examination of the factors in establishing the readiness of Kwekwe district for RBM as a teacher performance evaluation system.

\subsubsection{Pre-conditions to be ascertained through readiness assessment}

Existing literature emphasises the importance of conducting readiness assessment before introducing RBM (Mayne, 2007). Conducting readiness assessment assists the drivers of the system to time the introduction of a new performance evaluation system appropriately (Kusek \& Rist, 2004:40). Failure to appropriately time the introduction of an evaluation system may increase insecurity and fears on one hand or result in the process being overtaken by events on the other hand (Madhekeni, 2012; Robbins, 
Odendaal \& Roodt, 2003). Readiness assessment assists in providing early indicators of what to anticipate ahead of implementation and thus allow for the mapping up of relevant strategies.

In conducting readiness assessment, several factors such as the presence of a legal statute to support evaluation, political will, institutional capacity, fiscal resources availability, institutional culture, and demands for such a system should be considered (Kusek \& Rist, 2002; Kusek \& Rist, 2004; Lehman, Greener \& Simpson, 2002).

\subsection{Legal framework}

Readiness assessment includes establishing the existence of a legal framework upon which the evaluation process will be anchored. The legal parameters of the evaluation process can be derived from the constitutional law, statutory law, previous ruling precedencies from the courts, and negotiated agreements (Tucker \& DeSander, 2011). The important aspects of an evaluation system that include the evaluation criteria, procedures and consequences of the evaluation process should be hinged on legal principles for the system to be legally acceptable (Tucker \& DeSander, 2011). A legally defensible appraisal system should have policies that are written down, communicated and availed to all persons affected by them (Carroll \& Schneier, 1982). It should, however be noted that although the law or statute can be a useful way of legitimising a monitoring and evaluation system, on its own, it cannot guarantee that maximum effort will be directed towards development of a functional evaluation system (Mackay, 2006). This, therefore, justifies the need to explore other possible factors around the influence of timing in introducing an effective evaluation system.

\subsection{Impetus for an evaluation system}

In ascertaining readiness of an organisation for the planned change, it is prudent to establish whether there is impetus or demand for building a monitoring and evaluation system (Kusek \& Rist, 2004; Mackay, 2006). Observations show that negative responses to organisational changes are usually a result of the leaders overlooking the need to communicate the change message timeously and consistently (Armenakis \& Harris, 2002; Machingambi, 2013) to ascertain and create demand. Mackay (2006) asserts that meaningful demand is essential to institutionalise monitoring and evaluation (M \& E) successfully. It is, however, acknowledged that factors such as inadequate understanding of $\mathrm{M} \& \mathrm{E}$ functions and lack of experience with M \& E (Mackay, 2006) can hinder successful implementation of an evaluation system. Hale (2004) reiterates the importance of stakeholders having a clear understanding of the new system and an appreciation of its benefits. An appreciation of the benefits of the new system motivates the stakeholders to embrace the new performance evaluation system, whilst on the other hand, a lack of appreciation of the benefits of the change can lead stakeholders to perceive the initiative negatively by viewing it as mere additional stress (Ochurub et al., 2012).

Ochurub et al. (2012) further explain that the introduction of a new evaluation system could threaten the employees in terms of job security and their interests. It is therefore prudent to ensure that the employees understand the rationale behind the introduction of the appraisal system, the benefits of the system, the drivers of the system and the implementation process. This calls for aggressive marketing of the system to the relevant stakeholders to allow them to have a clear understanding of the mission, vision, strategies and outcomes of the new initiative (Gutuza, 2016; Madhekeni, 2012; Pretorius \& Ngwenya, 2008; Ochurub et al., 2012). The organisation, therefore, needs to build a convincing case for the change constituting a component of personal valence which addresses the benefits of the change to the stakeholders, thereby preventing the propagation of feelings of threat to self-interest (Armenakis \& Harris, 2002).

Armenakis and Harris (2002) suggest that organisational change like introducing a new evaluation system should be implemented in phases that overlap. The first of these phases would be the readiness phase that is characterised by preparing the stakeholders for change and garnering their support for the change. During the readiness phase, the change message should be directed at 
addressing issues of discrepancy and appropriateness. Discrepancy addresses the question of whether the change is needed, that is, creating demand. Weiner (2009) adds that in addressing the discrepancy, there is need to highlight the variation between the current and desired performance levels so as to foment dissatisfaction while at the same time creating an appealing vision of the future. This is done by convincing the stakeholders about the existence of a discrepancy and therefore the justification for the change to a new evaluation system. The change message should also convince the stakeholders of the appropriateness of the change. It should not be assumed that the existence of a discrepancy automatically makes the intended change appropriate but stakeholders may feel otherwise, and in that case, there will be need to convince them or else reconsider the change (Armenakis \& Harris, 2002).

Shinkfield and Stufflebeam (1995) suggest that before replacing or improving an evaluation system, there is need to secure the mandate from the appropriate constituency in the form of an agreement between the 'top down' administrative order and the 'bottom up' directive for the change to be acceptable. Ochurub et al. (2012) reiterate that the top down approach without the bottom-up support is a recipe for disaster. It has been observed that people are not enthusiastic about changes that they haven't contributed to, so stakeholder involvement from the planning process facilitates buyin, ownership and in turn readiness for the change (Shinkfield \& Stufflebeam, 1995). The stakeholders have to be encouraged to share and provide new ideas on introducing a new system. Involving the stakeholders helps to strengthen the support and mandate for change by earning participants' backing and commitment to the successful implementation of the new evaluation system. Involving the stakeholders from the onset, in diagnosing the situation, working on the strategy and structuring the decisions, fosters cohesion and promotes ownership of the change (Armenakis \& Harris, 2002).

Imposing an evaluation system leads to stakeholders not having a sense of ownership for the programme but rather, developing a negative attitude towards the system which in turn affects the outcomes of the system. Ochurub et al. (2012), therefore, recommends a stakeholder impact analysis, as part of the readiness assessment process, to address stakeholder concerns, challenges and assumptions before introducing a new evaluation system.

\subsection{Political will and commitment}

In conducting readiness assessment, there is need to ascertain whether there is a champion for the system. The champion should ideally be someone at the centre of policy making with influence on important decision-makers to increase the chances of acceptability by top management (Kusek \& Rist, 2004). Having a champion for the system helps solicit the buy-in, support and commitment of the top leadership (Ochurub et al., 2012). Although the availability of a champion is no guarantee that the evaluation system will be embraced, there have been cases of success, for example in Chile and Australia, and on the other hand, failure, as in the case of Egypt (Mackay, 2006).

Observations made in Zimbabwe were that the requisite political will was absent as even the top management (ministers, parliamentarians and even commissioners) had poor buy-in due to inadequate understanding and appreciation of results based management (Madhekeni, 2012). Leadership commitment is critical since they are the ones that authorise the use of requisite resources and allocate the time for implementation of the initiative (HRSA, n.d). Research shows that the effectiveness of an evaluation system is affected negatively by the absence of top leadership commitment (Mavhiki, Nyamwanza \& Dhoro, 2013; Musingafi, Dumbu \& Chadamoyo, 2013; Ochurub et al., 2012).

\subsection{Institutional capacity}

The process of readiness assessment should also include institutional capacity assessment in terms of availability of resources and expertise. Institutional capacity assessment should establish the organisation's ability to support the initiative with resources such as money, technology and consultation services, where necessary (HRSA, n.d). Sufficient human and material resources should be allocated to facilitate the change process. Availability of resources facilitate an adequate and smooth 
planning process. Financial resources are required to assist adequate marketing of the initiative by the champions and also to allow suitable training of the implementers so that they can cope with the new job requirement (Ochurub et al., 2012).

Many researchers have cited the unavailability of resources as having negatively impacted on RBM in Zimbabwe. Resource constraints have been blamed for inadequate pre-implementation training where most of the implementers failed to be trained (Gutuza, 2016; Machingambi, 2013; Madhekeni, 2012). Shinkfield and Stufflebeam (1995) note that, for a new programme to be successful, it is necessary to secure an appropriate level of financial and other resources. Besides preventing adequate marketing and pre-implementation training, the inadequacy of resources can affect other factors like procurement of the necessary material resources, provisions of incentives and funding for targeted staff development programmes, thereby, negatively affecting the effectiveness of the system. Ascertaining fiscal capacity of the organisation, therefore, helps to determine the appropriateness of the timing of introducing a new performance evaluation system.

\subsection{Organisational culture}

In conducting readiness assessment, it is also important to establish organisational culture for results. Organisational culture refers to the individual beliefs, perceptions and expectations that are shared by individuals within an organisation (HRSA, n.d). Results-oriented organisational culture is key as it can make or undo the successful implementation of the system by being an effective guide to appropriate and goal directed behaviours. Research suggest that negative organisational culture can hinder buy-in for a new system (Mavhiki et al., 2013; Mayne 2010). In the case of RBM, workers that are not resultsoriented will not be keen to account for their work contrary to RBM principles of accountability, transparency and demonstrable results. This would, therefore, call for a change of the mind-set of stakeholders by creating a new culture (Madhekeni, 2012). This can be done by having both management and stakeholders working together to create a culture of results-orientation (HRSA, n.d).

\subsection{Piloting the new evaluation system}

Armenakis and Harris (2002) suggest that after preparing the stakeholders for organisational change and winning their support, the next phase should be to adopt the change. This entails implementing the change on experimental basis where there is room for rejection or modification. A new system should be piloted prior to full scale implementation to provide for concerns and problems to be addressed. Soliciting stakeholder input during the piloting phase helps to earn their trust and increase their cooperation which enhances acceptability of the change (Shinkfield \& Stufflebeam, 1995).

In view of the above, the importance of readiness assessment cannot be overemphasised. Readiness assessment is key in ascertaining the appropriateness of introducing a new evaluation system. It determines the level of organisational preparedness and allows designers to assess the feasibility of the intended programme, thereby increasing the chances of its success and effectiveness. Overlooking this important step could lead to a waste of finances and other resources on systems that may be resisted and undermined by the implementers (Ochurub et al., 2012).

\section{Study Focus and Objectives}

The purpose of this study was to establish the influence that the timing of introducing a performance evaluation system has on effectiveness of the system. The influence of timing in introducing a performance evaluation system in relation to the effectiveness of the system was ascertained by determining the significance and strength of the relationship between the timing of introduction and effectiveness of the system. The rationale for the study was to determine how the different factors of the readiness assessment component of RADPS framework interacted to influence the effectiveness of RBM. This study will thus inform policy makers on factors that should be considered when deciding the timing of introducing a new performance evaluation system to enhance the effectiveness of the system. 


\subsection{Study hypotheses and assumptions}

In assessing the effect of timing of introducing a teacher performance evaluation (TPE) on the effectiveness of the system, we had the following two hypotheses and one assumption:

- There is no significant relationship between the timing of introducing TPE and its intended outcomes.

- There is no strong relationship between the timing of introducing TPE and its impact on achievement of its intended outcomes.

Since TPE has been introduced for over five years in the district where the study was conducted, we assumed that there was an assessment of readiness to introduce the evaluation system before its inception in terms of legal statute, demand, institutional capacity, political will, organisational culture and piloting.

\section{Methodology}

\subsection{The Research Paradigm}

The study was premised on the pragmatic worldview which accepts both the observable and relative knowledge (Morgan, 2014). The pragmatic approach rejects methodological dogmatism and focuses on what works in relation to tackling the presenting problem (Creswell, 2014).

\subsection{The Research Design}

The convergent parallel mixed method design was used. Quantitative and qualitative data were collected and analysed separately but concurrently (Creswell, 2014; Creswell, 2015; Teddlie \& Tashakkori, 2009) as illustrated in Figure 1.

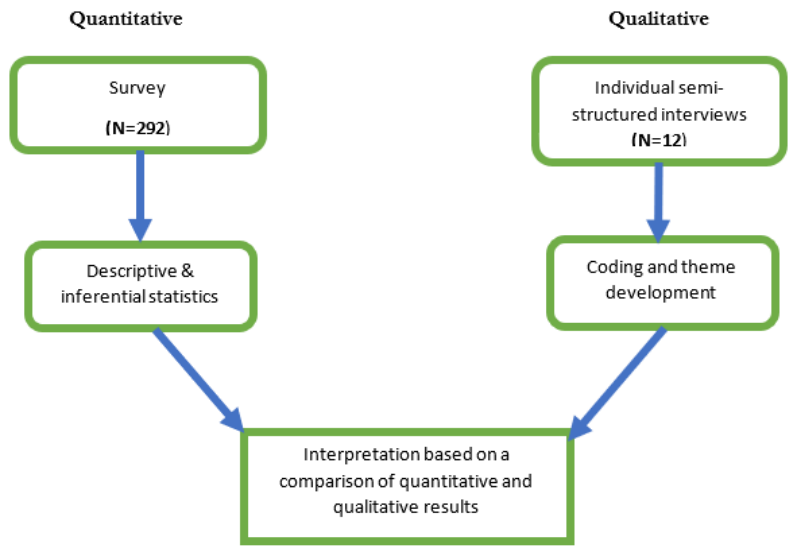

Figure 1: Convergent parallel mixed methods design procedures

Source: Adapted from: Ivankova, Creswell \& Plano Clark, 2016

\subsection{The Sampling Process}

The participating schools were selected using the stratified purposive sampling technique following defined criteria to derive rural and urban schools' strata. Stratified sampling entailed the researchers identifying subgroups or strata in the population so that each unit belonged to a particular stratum (Teddlie \& Tashakkori, 2009). The defining criteria for the schools was that the rural schools be located at least $45 \mathrm{~km}$ from a town centre, in areas under the authority of a rural district council while urban 
schools were located in an area under the control of an urban municipality council. A total of ten schools constituting of 5 urban and 5 rural secondary schools which had conducted performance management evaluation were selected to participate in the survey, while from the ten, 2 urban and 2 rural schools were purposively selected for the interviews. In selecting the study participants, the concurrent parallel sampling design was used. The samples for the two strands of the study were selected simultaneously but had a parallel relationship. This meant that the quantitative and qualitative samples were different but drawn from the same underlying population (Collins, Onwuegbuzie \& Jiao, 2007). The simple random sampling technique was used to select 292 respondents for the survey. The stratified purposive sampling technique guided by a defining criterion was used to select 12 key informants comprising of 4 heads of schools, 4 heads of departments and 4 teachers for the interviews. Educators for the interviews were selected on the basis that they were already in service when the RBM was adopted by the government.

\subsection{Data Analysis}

SPSS version 26 was used to produce frequency/percentage tables and to conduct the Analysis of Variance (ANOVA), and Beta coefficient tests (multiple regression analysis). The qualitative analysis process was conducted using ATLAS ti. 8 to produce quotations and network views.

\section{Results}

The study established a number of factors suggestive of mistiming of introduction of RBM as a teacher performance evaluation system. These include lack of knowledge of the evaluation regulations, imposition of the system, inadequate impetus, lack of buy-in, inadequate pre-implementation training, and inadequate resources.

\subsection{Lack of knowledge of performance evaluation regulations}

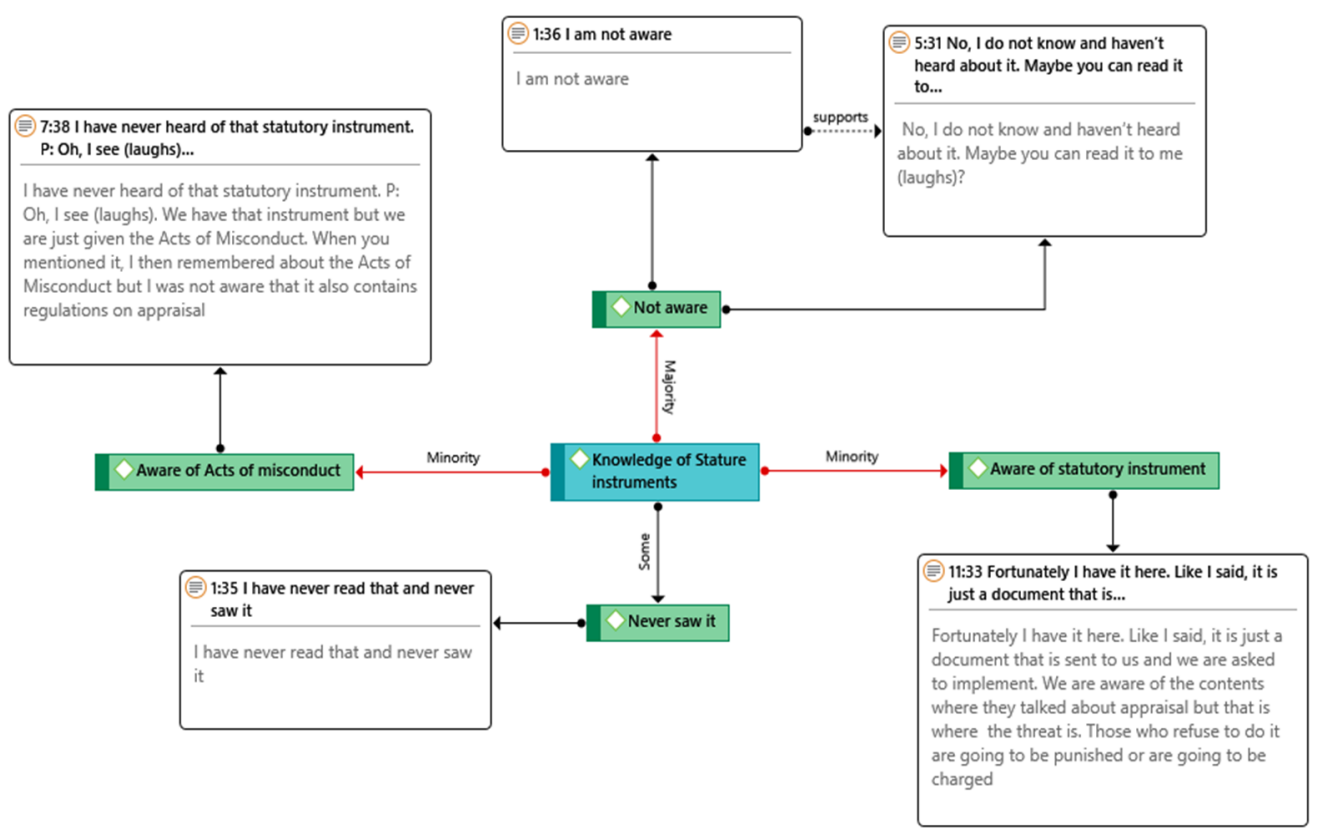

Figure 2: Knowledge of the legal statute on the performance evaluation process. 
The study established that the evaluation process is anchored on Statutory Instrument 1 of 2000 of the Government of Zimbabwe (Public Service Commission (Zimbabwe), 200o). The interesting aspect, however, is that, although the evaluation process is guided by the Statutory 1 of 2000 , the majority of the participants were not aware of it as shown in Figure 2. Eight out of the 12 participants that were interviewed were not aware of the statutory instrument. This was confirmed by some of the comments like, 'I am not aware' (Teacher 2). This was supported by another participant who said, "No, I do not know and haven't heard about it. Maybe you can read it to me..." (HOD 3). Most of the participants expressed similar sentiments. The instrument contains regulations on many aspects but it appears that although some participants knew of the Acts of Misconduct that are in the same instrument, they were however not privy to the appraisal regulations. This was highlighted by a participant who said, “...We have that instrument but we are just given the Acts of Misconduct. When you mentioned it, I then remembered about the Acts of Misconduct but I was not aware that it also contains regulations on appraisal" (Teacher 4). This raises eyebrows on whether these regulations are enforced after all.

Questions in Table 1 were used to find out the teachers' perceptions on the appropriateness of the timing of introduction of the evaluation system while Figure 3 presents a summative picture of the different concerns regarding the timing of introduction raised by the 12 participants of the interviews. Since the study intended to corroborate the quantitative and qualitative findings, the results were thus compared for either convergence or divergence.

Table 1: Perceptions of teachers on the appropriateness of the timing of introduction of the teacher evaluation system

\begin{tabular}{|c|c|c|c|c|c|}
\hline & \multicolumn{5}{|c|}{ Frequency /Percentage } \\
\hline & S D & D & NS & $\mathbf{A}$ & $\mathbf{S} \mathbf{A}$ \\
\hline $\begin{array}{l}\text { There was adequate consultation before inception of } \\
\text { Results Based Management }\end{array}$ & $\begin{array}{c}86 \\
29 \cdot 5 \%\end{array}$ & $\begin{array}{c}73 \\
25 \%\end{array}$ & $\begin{array}{c}74 \\
25 \cdot 3 \%\end{array}$ & $\begin{array}{c}55 \\
18.8 \%\end{array}$ & $\begin{array}{c}4 \\
1.4 \%\end{array}$ \\
\hline $\begin{array}{l}\text { I was trained on Results Based Management System } \\
\text { (RBM) before it was introduced. }\end{array}$ & $\begin{array}{c}76 \\
26 \% \\
\end{array}$ & $\begin{array}{c}87 \\
29.8 \% \\
\end{array}$ & $\begin{array}{c}22 \\
7 \cdot 5 \% \\
\end{array}$ & $\begin{array}{c}99 \\
33 \cdot 9 \% \\
\end{array}$ & $\begin{array}{c}8 \\
2.7 \% \\
\end{array}$ \\
\hline I was ready for RBM when it was introduced. & $\begin{array}{c}84 \\
28.8 \% \\
\end{array}$ & $\begin{array}{r}119 \\
40.8 \% \\
\end{array}$ & $\begin{array}{c}35 \\
12 \% \\
\end{array}$ & $\begin{array}{r}48 \\
16.4 \% \\
\end{array}$ & $\begin{array}{c}6 \\
2.1 \% \\
\end{array}$ \\
\hline I knew all the requirements for RBM. & $\begin{array}{c}78 \\
26.7 \%\end{array}$ & $\begin{array}{c}113 \\
38.7 \%\end{array}$ & $\begin{array}{c}51 \\
17.1 \%\end{array}$ & $\begin{array}{c}41 \\
14 \%\end{array}$ & $\begin{array}{c}9 \\
3.1 \%\end{array}$ \\
\hline $\begin{array}{l}\text { My school had all the necessary requirements for RBM } \\
\text { when it was introduced. }\end{array}$ & $\begin{array}{c}58 \\
19.9 \% \\
\end{array}$ & $\begin{array}{c}87 \\
29.8 \% \\
\end{array}$ & $\begin{array}{r}84 \\
28.8 \% \\
\end{array}$ & $\begin{array}{c}48 \\
16.4 \% \\
\end{array}$ & $\begin{array}{c}9 \\
3.1 \% \\
\end{array}$ \\
\hline $\begin{array}{l}\text { When RBM was introduced, I was not sure of what was } \\
\text { expected of me }\end{array}$ & $\begin{array}{c}46 \\
15.8 \% \\
\end{array}$ & $\begin{array}{r}37 \\
12.7 \% \\
\end{array}$ & $\begin{array}{c}30 \\
10.3 \% \\
\end{array}$ & $\begin{array}{c}45 \\
15 \cdot 4 \% \\
\end{array}$ & $\begin{array}{c}134 \\
45.9 \% \\
\end{array}$ \\
\hline $\begin{array}{l}\text { RBM was needed in the education system to appraise } \\
\text { teacher performance. }\end{array}$ & $\begin{array}{c}61 \\
20.9 \% \\
\end{array}$ & $\begin{array}{c}49 \\
16.8 \% \\
\end{array}$ & $\begin{array}{c}63 \\
21.6 \% \\
\end{array}$ & $\begin{array}{r}92 \\
31.5 \% \\
\end{array}$ & $\begin{array}{c}27 \\
9.2 \% \\
\end{array}$ \\
\hline $\begin{array}{l}\text { My school head as the manager supported the } \\
\text { Introduction of RBM as an appraisal system. }\end{array}$ & $\begin{array}{c}18 \\
6.2 \% \\
\end{array}$ & $\begin{array}{c}18 \\
6.2 \% \\
\end{array}$ & $\begin{array}{c}69 \\
23.6 \% \\
\end{array}$ & $\begin{array}{c}143 \\
49 \% \\
\end{array}$ & $\begin{array}{c}44 \\
15.1 \% \\
\end{array}$ \\
\hline For me, the introduction of $\mathrm{RBM}$ was at the right time. & $\begin{array}{c}69 \\
23.6 \% \\
\end{array}$ & $\begin{array}{c}98 \\
33.6 \% \\
\end{array}$ & $\begin{array}{c}69 \\
23.6 \% \\
\end{array}$ & $\begin{array}{c}48 \\
16.4 \% \\
\end{array}$ & $\begin{array}{c}8 \\
\mathbf{2 . 7} \% \\
\end{array}$ \\
\hline $\begin{array}{l}\text { My opinion is that the timing of the inception of the } \\
\text { teacher evaluation system has impacted positively on } \\
\text { the teacher performance evaluation effectiveness. }\end{array}$ & $\begin{array}{c}73 \\
25 \%\end{array}$ & $\begin{array}{c}77 \\
26.4 \%\end{array}$ & $\begin{array}{c}73 \\
25 \%\end{array}$ & $\begin{array}{c}63 \\
21.6 \%\end{array}$ & $\begin{array}{c}6 \\
2.1 \%\end{array}$ \\
\hline
\end{tabular}

SD-Strongly Disagree D-Disagree NS-Not Sure A-Agree SA-Strongly Agree




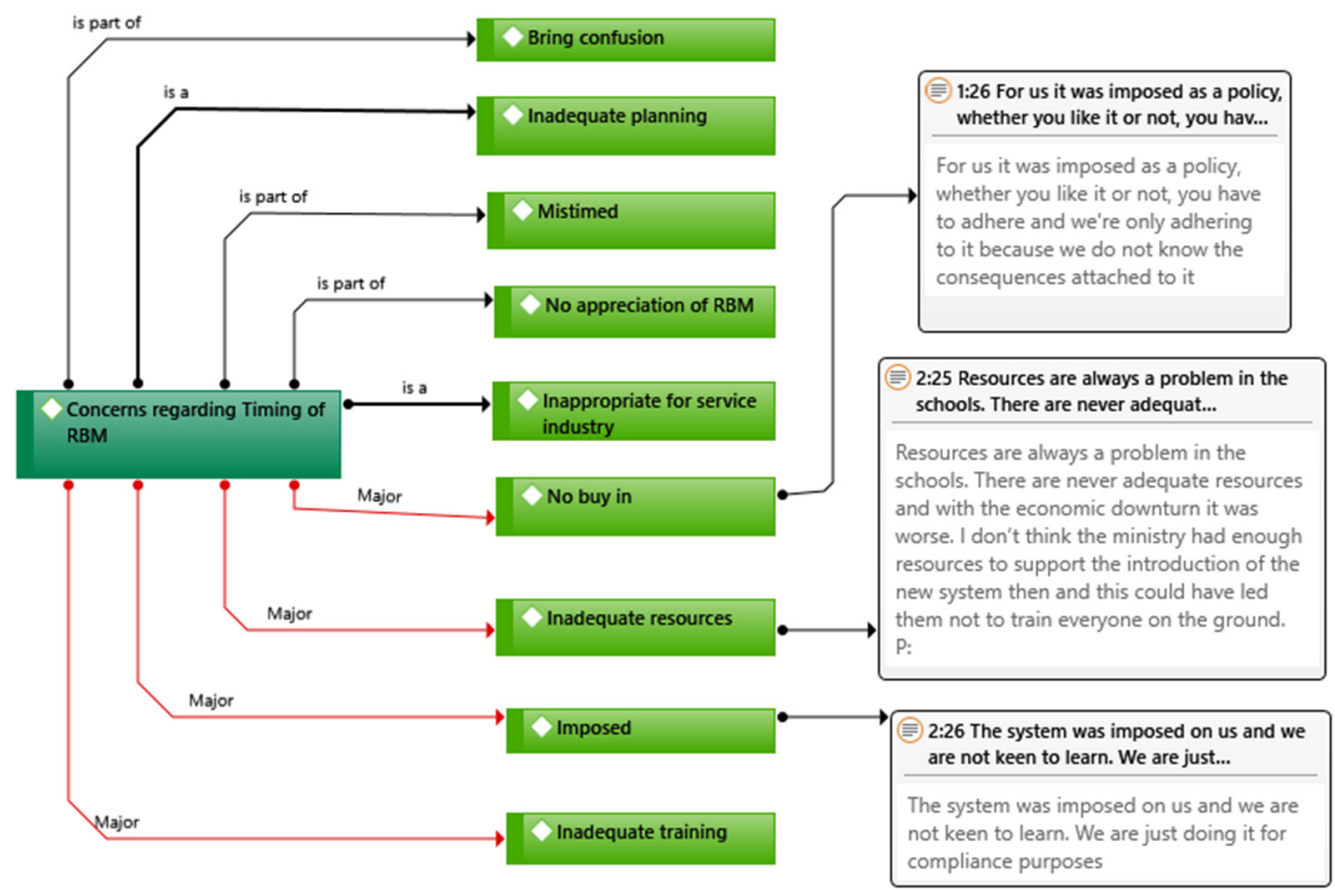

Figure 3: Concerns regarding the timing of the programme

\subsection{Imposition of the evaluation system}

Indications from quantitative data in table 1 were that the RBM system was introduced as a teacher performance evaluation system without much having been done in terms of consulting the teachers. From the 292 teachers who responded to the questionnaire, $159(54.5 \%)$ pointed out that there had been inadequate consultations before the introduction of RBM. This position was ratified by participants from the interviews (Figure 3), with eight of them confirming that no consultations had been made prior to the introduction of the system. This was reflected in some of the participants' comments that, "I personally feel that this was imposed on us. There wasn't any dialogue" (HOD 1). In support of this, another participant echoed that, "The system was imposed on us and we are not keen to learn. We are just doing this for compliance purposes” (Teacher 2).

\subsection{Inadequate impetus for RBM}

This study established that there was no adequate impetus for RBM from the majority of the stakeholders. Data in table 1 shows that there was no adequate demand for the evaluation system as only $40.7 \%$ of the teachers affirmed that RBM was needed to appraise teachers while on the other hand $37.7 \%$ of the respondents disagreed that RBM was needed to appraise teacher performance. This position was corroborated by the majority of the participants testifying that RBM does not have benefits to the teacher nor the learner. Most of the participants felt that RBM only benefits the administrators. One of the participants testified, "But for the teachers ..., I do not think there is any benefit besides stress" (Teacher 3). Another teacher added, "It isn't benefitting the learner. Actually it's wasting time, because the time when the teacher has to make sure that the form has been completed, nothing will be happening in the classroom" (Teacher 1). 


\subsection{Lack of buy-in}

The fact that only a total of $40.7 \%$ of the respondents agreed that RBM was needed in the education system to appraise teacher performance is an indication that there was no buy-in nor acceptance by the implementers. Findings from the interviews also validated this point as reflected by some of the comments by the participants that, "There was need for the ministry to assess if the stakeholders embraced the system before introducing it." (Teacher 3). Implications of such sentiments were that the system was not accepted or embraced because it had been imposed on the teachers. A head of department also explained that. "...embracing that system might be very difficult especially if you do not know what you are supposed to be doing" (HOD 4). The ultimate effect of the top-down approach was that there was neither buy-in nor ownership of the system by the supposed implementers. It can also be deduced from this statement that lack of buy-in could have been caused by inadequate understanding of the system and the implementation process. Another participant further added that, "I also feel that RBM is more suited to the manufacturing and industrial sector than it is to the service industry like education" ( $\mathrm{HOD}_{3}$ ). This statement suggests lack of conviction by the participant on the suitability of RBM as a teacher performance evaluation system implying that there was inadequate marketing prior to introducing the system.

\subsection{Inadequate pre-implementation training}

It also emerged from the study that the pre-implementation training had been inadequate. Indications by $163(55.8 \%)$ respondents were that they had not received any pre-implementation training on the system before its introduction. Only $36.6 \%$ of the teachers attested that they had received preimplementation training. The stance that pre-implementation training was inadequate was corroborated by the majority of the interview participants. The failure by the programme drivers to provide adequate pre-implementation training is blamed for the inability of the majority of the teachers to implement the system. This was revealed in the quotations by some of the participants: "We didn't actually know what was expected of us...only a few people were coached and when they came to the schools, they didn't actually understand what they had learnt. We were all confused about what we were supposed to do, so we were simply copying and pasting from each other" (Teacher 2). Likewise, another teacher also indicated that, "We did not know exactly what it was all about and sometimes we get confused" (Teacher 3). This was reiterated by a head of department who further explained that, "They did not have much information, although workshops were held but then people were still confused" (HOD $2)$. This was further affirmed by $179(61.39 \%)$ of the respondents acknowledging that when RBM was introduced, they did not know what was expected of them. A total of 191 (65.4\%) respondents further supported this position when they refuted that they knew all requirements for RBM.

These sentiments of the participants were indicative of the inadequacy of the pre-implementation training. Research findings suggest that the pre-implementation training was too deficient to equip the teachers with the requisite knowledge to implement the system. The interviewees also indicated that the majority of individuals that had been trained had limited knowledge and understanding of the system yet they were expected to train others. This resulted in them providing sub-standard training which failed to demystify the new system, but instead, only brought more confusion to the teachers. Most of the teachers, therefore, ended up just copying from the few who had limited knowledge in order to comply with the policy requirements.

\subsection{Inadequate resources to support the system}

Another sentiment from the interview participants was that resources were inadequate to support the introduction of a new performance evaluation system then. Statements from some of the participants like, “...I don't think the ministry had enough resources to support the introduction of the new system then and this could have led them not to train everyone on the ground" (Teacher 1). Deductions that can be made 
from this statement are that the organisation was not financially capacitated to introduce the new system then. Fiscal space was a necessary prerequisite for the pre-implementation training, funding of targeted staff development programmes and provision of incentives. The failure to provide adequate training is therefore blamed on the lack of adequate fiscal support for the programme. The same teacher went further to explain that, “...There was never adequate resources and with the economic downturn, it was made worse" (Teacher 1). The suggestion by the statement is that introducing the new system at that time was thus inappropriate. These claims were confirmed by 167 (57.2\%) teachers who refuted that RMB had been introduced at the right time. This was further supported by 150 (51.4\%) teachers who repudiated that the timing of the inception of the teacher performance evaluation system had impacted positively on the effectiveness of the system. Findings from the survey and interviews were confirmed by the results of inferential statistics (ANOVA and Beta coefficient tests) that were conducted.

\subsection{ANOVA Test on significance of relationship of timing of introduction and effectiveness of RBM}

The ANOVA test showed acceptability of the regression model from a statistical perspective and reported how well the regression equation fitted the data. The ANOVA results for this study are presented in Table 2.

Table 2: ANOVA table

\begin{tabular}{|c|c|c|c|c|c|c|}
\hline \multicolumn{7}{|c|}{ ANOVA $^{a}$} \\
\hline Mode & & Sum of Squares & $\mathrm{df}$ & Mean Square & $\mathrm{F}$ & Sig. \\
\hline \multirow{3}{*}{1} & Regression & 103.249 & 4 & 25.812 & 35187.882 & $.000^{\mathrm{b}}$ \\
\hline & Residual & .211 & 287 & .001 & & \\
\hline & Total & 103.460 & 291 & & & \\
\hline \multicolumn{7}{|c|}{ a. Dependent Variable: Effectiveness } \\
\hline
\end{tabular}

The regression row shows information about the variation accounted for by the model while the residual row shows information about the variation not accounted for by the model. The regression sum of squares (103.249) is considerably larger than the residual sum of squares (.211) indicating that most of the variation is explained by the model thereby making the model fit for the analysis of the data. The high regression and consequently low residual values indicate that the explanation from the model is not due to chance. The significant value of o.ooo means that there is a statistically significant relationship between the timing of introducing and the effectiveness of the system, in other words, timing is statistically significant in predicting the effectiveness of teacher performance evaluation systems. We thus found a statistically significant positive association between timing of introduction and effectiveness of a programme.

\subsection{The Beta coefficient test on strength of influence of timing on effectiveness of RBM}

The Beta coefficient test which explains the strength of the relationship between the independent variable and the dependent variable was also conducted. In this study, it explains the extent of the influence of timing on the effectiveness of the teacher performance evaluation system. The results of the Beta coefficient test are presented in Table 3.

Table 3: Beta Coefficient table

\begin{tabular}{|c|c|c|c|c|c|c|}
\hline \multicolumn{7}{|c|}{ Coefficients $^{\mathrm{a}}$} \\
\hline \multirow{2}{*}{\multicolumn{2}{|c|}{ Model }} & \multicolumn{2}{|c|}{ Unstandardized Coefficients } & \multirow{2}{*}{$\frac{\text { Standardized Coefficients }}{\text { Beta }}$} & \multirow{2}{*}{$\mathrm{T}$} & \multirow{2}{*}{ Sig. } \\
\hline & & $\mathrm{B}$ & Std. Error & & & \\
\hline \multirow{2}{*}{1} & (Constant) & -.016 & .009 & & -1.709 & .089 \\
\hline & Timing & .186 & .003 & .213 & 60.308 & .000 \\
\hline
\end{tabular}


We used the standardised Beta coefficient as we had standardised the variables by transforming the data from categorical scale to continuous scale before running the regression analysis. The results in this table show that timing has a beta coefficient value of -213 thereby proving that there is a relatively strong relationship between timing of introducing an evaluation system and its effectiveness. Results from the ANOVA and Beta coefficient tests therefore confirm the findings from the survey and interviews which explain the existence of a relationship between the timing of introduction and the effectiveness of a performance evaluation system.

\section{Discussion of Results}

A number of facts emerged from this study relating to the timing of the introduction of RBM as a teacher performance appraisal system. The main issues that were highlighted include limited knowledge of the statute on evaluation by the implementers, imposition by the authorities, inadequate resources, insufficient marketing of the system, inadequate training and lack of buy-in.

\subsection{Limited knowledge of a legal statute}

According to Tucker and Desander (2011), it is important to have a legally defensible evaluation system which is premised on legal principles. The findings from the study show that although there is a legal framework underpinning the evaluation process, the majority of the teachers, including some HODs, are not even aware of the existence or contents of the statute. The findings show that Kwekwe district fails to satisfy the propositions by the Readiness Assessment of the RADPS conceptual framework that proposes for the evaluation regulations to be availed to all stakeholders and to be enforceable. Having the written regulations which are neither known nor availed to the relevant stakeholders tends to make the evaluation process a rhetoric exercise. The fact that the regulations are not known implies that there may have been inadequate communication to the teachers prior to introducing the system. If the teachers had been adequately informed about the system, then, they should have been cognisant of the evaluation regulations. The fact that the regulations are not known by most of the teachers, therefore, suggests that the system was introduced before adequate ground work had been done, thereby making it mistimed. This is contrary to the suggestions by Carroll \& Schneier (1982) that all policies guiding the appraisal system should be communicated and availed to all persons affected by them.

\subsection{Imposition of performance evaluation on teachers}

Findings from the study show that the performance evaluation system was imposed on the implementers. The drivers of the process failed to seek the mandate from the key stakeholders (teachers) as suggested in literature (Ochurub et al., 2012; Shinkfield \& Stufflebeam, 1995). This deprived the process of the requisite support and commitment by the implementers which is vital for effective change. The lack of involvement of the key stakes contributed to the lack of buy-in and acceptance of the system by the teachers (Gutuza, 2016). The failure to consult and involve the stakeholders suggest premature introduction of the system before adequate marketing, thereby hindering buy-in and ownership of the system by the teachers. Evidence from the study suggest that most teachers were yet to embrace the system years after it had been adopted and were just implementing it for policy compliance purposes, without appreciating its utility and value. This falls short of the attribute of utility which asserts that the stakeholder need to appreciate the usefulness of an evaluation process for them to respect it (Stufflebeam \& Shinkfield, 1995). The need to market a programme to the implementers for them to be conscious and appreciative of its benefits is recommended for the promotion of buy-in and acceptance, (Armenakis \& Harris, 2002; Pretorius \& Ngwenya, 2008). Introducing RBM without active involvement or consultation of the teachers has led to the system not getting accepted by the teachers, thereby suggesting that the inception was mistimed. 


\subsection{Inadequate buy-in}

The lack of buy-in and ownership of the system by the stakeholders has also been ascribed to inadequate stakeholders' involvement in the case of Zimbabwe (Gutuza, 2016; Machingambi, 2013; Shinkfield \& Stufflebeam, 1995). Gutuza (2016) adds that lack of involvement of stakeholders propagate resistance and negative regard for the system. The involvement of teachers and/or their unions on the other hand can minimise teacher resistance and instead promote their buy-in as it happened in the case of Chile (Santiago \& Benavides, 2009). The Chilean teacher evaluation system was designed in a participatory way with an active engagement and this helped to enhance its acceptance and resultantly its effectiveness. It is, therefore, prudent to ensure that the timing of introducing a new performance evaluation system is not rushed before stakeholder buy-in has been established, otherwise, effectiveness of the system is hampered.

\subsection{Impetus for RBM}

The RADPS conceptual framework proposes that readiness assessment should be conducted to determine the demand for the programme which in turn helps to guide in the appropriate timing of introducing a new intervention like RBM. Findings from this study have confirmed the importance of readiness assessment to determine the timing of introducing a programme. Findings show that there was no impetus for RBM when it was introduced. Mackay (2006) emphasises the need to create demand for the successful institutionalisation of an evaluation system. The marketing of an evaluation system can help create demand for it when stakeholders are made aware of the rationale for the change and the benefits of the process (Gutuza, 2016; Madhekeni, 2012; Pretorius \& Ngwenya, 2008; Ochurub et $\mathrm{al}, 2012$ ). Marketing leads to knowledge building and appreciation of the new intervention by the implementers. Marketing also creates an opportunity for dialogue, thereby demystifying the grey areas of the new product and in turn promoting acceptance. The findings from the study therefore resonate with the propositions of the RADPS conceptual framework to ascertain preparedness for a programme before introducing it to increasing the chances of successful implementation.

\subsection{Inadequate resources}

This study established that there were inadequate resources to support the introduction of RBM. Other studies also highlighted resource constraints as one of the factors that hindered successful implementation of RBM (Gutuza, 2016; Machingambi, 2013; Madhekeni, 2012). Existing gaps need to be identified to build and introduce a sustainable evaluation system (Kusek \& Rist 2004). Making budgetary considerations is key as it helps to ensure that the introduction of the new intervention is not mistimed and thus likely to stall (Shinkfield \& Stufflebeam, 1995). Readiness assessment as proposed by the RADPS conceptual framework helps to establish the adequacy of the resources required to introduce the new system and thus ascertain the feasibility of the intended programme.

\subsection{Inadequate pre-implementation training}

Interviewees acknowledged that they were confused and did not understand much about how the RBM was supposed to be implemented. Findings by Machingambi (2013) and Gutuza (2016) also confirmed that teachers were not capacitated and lacked the requisite skills to implement the evaluation system properly. Such lack of capacitation of the teachers was attributed to the inadequate preimplementation training that had been provided. The quality of pre-training given could possibly have been compromised by the limited availability of resources to support the training agenda. As proposed by the RADPS conceptual framework and extant literature, this study confirms the importance of readiness assessment so as to determine an appropriate time to introduce a new intervention such as RBM. 


\section{Conclusion}

Based on the findings made, this study concludes that the timing of the introduction of RBM as a teacher evaluation system was inappropriate. Indications are that the introduction of the system was mistimed in view of the fact that the drivers of the programme had not acquired adequate resources and funding to support the introduction of the new system. This in turn compromised the champions' ability to market the new system to the stakeholders for them to appreciate the benefits and also limited the number of training workshops and teachers involved which in turn hampered the ability of teachers to implement. Evidence suggest that the system was introduced prematurely without adequate planning and preparation. It was, therefore, concluded that the timing of the introduction of RBM negatively affected its effectiveness as a teacher performance evaluation system.

The study established that there was no marketing nor consultations of the stakeholders prior to the introduction of the system. This, coupled with the inadequate involvement of the teachers and inadequate pre-implementation training led the implementers to have limited buy-in. Participants ascribed the lack of marketing and inadequate training to the existence of limited budgetary support for the new programme. Taking into account the findings and resultant conclusions made therein, this study recommends that readiness assessment should be conducted prior to introducing a new programme to ascertain its feasibility and viability. The Readiness Assessment component of the RADPS conceptual framework developed by this study explains a set of factors that contribute to ensuring preparedness for a new system and thus help to determine the appropriateness of introducing a new performance evaluation system.

\section{Acknowledgements}

This paper is part of a bigger study that was submitted to a university for a doctoral thesis.

\section{Reference}

Armenakis, A. A., \& Harris, S. G. (2002). Crafting a change message to create transformational readiness. Journal of Organizational Change Management, 15, 169-183.

Carroll, S.J., Schneier, C.E. (1982). Performance Appraisal and Review Systems: The Identification. Measurement and Development of Performance in Organisations, Scott Foresman, Glenview, IL.

Collins, K. M., Onwuegbuzie, A. J., \& Jiao, Q. G. (2007). A mixed methods investigation of mixed methods sampling designs in social and health science research. Journal of mixed methods research, 1(3), 267-294.

Creswell, J. W. (2014). Research design: qualitative, quantitative, and mixed methods approaches. (4 ${ }^{\text {th }}$ ed.). Thousand Oaks: Sage Publications, Inc.

Creswell, J. W. (2014). A concise introduction to mixed methods research. Thousand Oaks: Sage Publications, Inc.

Darling-Hammond, L. (2000). Teacher quality and student achievement: A review of state policy evidence. Education Policy Analysis, 8(1), 15-17.

Dorsey, D. \& Mueller-Hanson, R. (n.d). Performance management that makes a difference: An evidence-based approach. [Online] Available: https://www.shrm.org/hr-today/trends-and-forecasting/special-reports-andexpert-views/documents/performance\%2omanagement.pdf (February 18, 2020)

Elliott, K. (2015). Teacher Performance Appraisal: More about Performance or Development? Australian Journal of teacher education, $40(9)$, 9 .

Gutuza, R.F. (2016). An investigation on the challenges faced by schools in implementation of the Result Based Management system in Mutasa district of Zimbabwe. Global Journal of Advanced Research, 3(5), 389-396.

Health Resources and Services Administration. (n.d.). Readiness Assessment and developing project aims. [Online] Available:https://www.hrsa.gov/sites/default/files/quality/toolbox/508pdfs/readinessassessment.pdf

Kusek, J. Z., \& Rist, R. C. (2002). Building results-based monitoring and evaluation systems: Assessing developing countries readiness. Zeitschrift für Evaluation, 1, 151-58.

Kusek, J.Z. \& Rist, R.C. (2004). Ten steps to a results-based monitoring and evaluation system: a handbook for development practitioners. [Online] Available: https://openknowledge.worldbank.org/bitstream/handle /10986/14926/29672oPAPERo1oosteps.pdf (December 19, 2017) 
Lehman, W. E., Greener, J. M., \& Simpson, D. D. (2002). Assessing organizational readiness for change. Journal of substance abuse treatment, 22(4), 197-209.

Looney, J. (2011). Developing high-quality teachers: Teacher evaluation for improvement. European Journal of Education, 46(4), 440-455.

Ivankova, N.V., Creswell, J. W., \& Plano Clark, V. L. (2016). Foundations and approaches to mixed method research. In K, Maree, (Ed), First steps in research 2 (pp.305-336). Pretoria: Van Schaik Publishers.

Machingambi, S. (2013). Teachers' perceptions on the implementation of the performance management system in Zimbabwe. International Journal of Educational Sciences, 5(3), 217-225.

Mackay, K. (2006). Institutionalization of monitoring and evaluation systems to improve public sector management. [Online] Available: http://siteresources.worldbank.org/INTEVACAPDEV/Resources/45856641254406777526/monitoring_evaluation_psm.pdf(December 22, 2017).

Madhekeni, A. (2012). Implementing results-based management systems in Zimbabwe: Context and implications for the public sector. International Journal of Humanities and Social Science, 2(8), 122-129.

Matunhu, J., \& Matunhu, V. (2014). A Review of the Implementation of Public Service Reforms by Zimbabwe Revenue Authority (2011). Journal of Social Sciences, 38(1), 63-70.

Mavhiki, S., Nyamwanza, T., \& Dhoro, L. (2013). An evaluation of RBM implementation in the civil service sector in Zimbabwe. European Journal of Business and Management, 5(32), 135-139.

Mayne, J. (2007). Challenges and lessons in implementing results-based management. Evaluation, 13(1), 87-109.

Mayne, J. (2010). Building an Evaluative culture: The key to effective evaluation and results management. The Canadian Journal of Program Evaluation, 24(2), 1-30.

Morgan, D.L. (2014). Integrating qualitative and quantitative methods: A pragmatic approach. Thousand Oaks: Sage Publications, Inc.

Musingafi, M. C., Dumbu, E. (2013). Improving performance in the African Civil Service: Empirical Evidence from Zimbabwe. Public Policy and Administration Research, 3(2), 19-26.

Ochurub, M., Bussin, M., \& Goosen, X. (2012). Organisational readiness for introducing a performance management system. SA Journal of Human Resource Management, 10(1), 1-11.

Pretorius, S. G., \& Ngwenya, V. C. (2008). Teachers' perceptions of and attitudes towards performance appraisal in Zimbabwean schools. Africa Education Review, 5(1), 144-164.

Public Service Commission. (2000). Public Service Regulations: Statutory Instrument 1 of 2ooo. Harare: Government Printers.

Sanders, W. L., Wright, S. P., \& Horn, S. P. (1997). Teacher and classroom context effects on student achievement: Implications for teacher evaluation. Journal of personnel evaluation in education, 11(1), 57-67.

Santiago, P., \& Benavides, F. (2009, December 1-2)). Teacher evaluation: A conceptual framework and examples of country practices. [Paper for presentation]. Towards a teacher evaluation framework in Mexico: International Practices, Criteria and Mechanisms, Mexico City, Mexico. http://www.oecd.org/edu/school/44568106.pdf

Shinkfield, A. J. \& Stufflebeam, D.L. (1995). Teacher Evaluation: Guide to Effective Practice. Boston: Kluwer Academic Publishers. [Online] Available: http://files.eric.ed.gov/fulltext/ED435632.pdf [September 17, 2017]

Stronge, J.H. \& Tucker, P.D. (2003). Handbook on Teacher Evaluation: Assessing and Improving Performance. Larchmont, NY: Eye On Education.

Stronge, J. H. (1995). Balancing individual and institutional goals in educational personnel evaluation: A conceptual framework. Studies in Educational Evaluation, 21(2), 131-151.

Teddlie, C., \& Tashakkori, A. (2009). Foundations of mixed methods research: Integrating quantitative and qualitative approaches in the social and behavioral sciences. Thousand Oaks: Sage Publications, Inc.

The New Teacher Project. (2010). Evaluation 2.0 [Online] Available: www.tntp.org/assets/documents/TeacherEvaluation-OctioF.pdf [October 18, 2018].

Tucker, P.D., \& DeSander, M.K. (2011). Legal Considerations in Designing Teacher Evaluation Systems. In J.H. Stronge (Ed), Evaluating Teaching (pp.69-98). Thousand Oaks: SAGE Publications, Inc.

UNDG. (2011). Results-Based Management Handbook. United Nations Development Group.

UNESCO. (2015). Results-based programming, management, monitoring and reporting (RBM) approach as applied at UNESCO: Guiding principles [Online] Available: http://unesdoc.unesco.org/images/oo17/oo1775/177568E.pdf [December 18, 2018]

Verspoor, A. M. (2004). The quest for quality: Towards a learning community. ADEA Newsletter, $16(1)$, 5-8.

Weiner, B. J. (2009). A theory of organizational readiness for change. Implementation science, 4(1), 67.

Zvavahera, P. (2014). An evaluation of the effectiveness of performance management systems on service delivery in the Zimbabwean civil service. Journal of Management and Marketing Research, 14, 1-8. 